\title{
Identification of Uncomplicated Patients With Acute Myocardial Infarction Undergoing Percutaneous Coronary Intervention
}

\author{
Are These Patients Suitable \\ for Early Discharge?
}

\author{
Kunihiro Kinjo, MD; Hiroshi Sato, MD; Yasuhiko Sakata, MD; Daisaku Nakatani, MD; \\ Hiroya Mizuno, MD; Masahiko Shimizu, MD; Masami Nishino, MD*; \\ Yasuhiko Matsu-ura, MD**; Yukihiro Koretsune, $\mathrm{MD}^{\dagger}$; Shinsuke Nanto, MD ${ }^{\dagger+}$; \\ Masayoshi Mishima, MD ; Masatsugu Hori, MD; on Behalf of \\ the Osaka Acute Coronary Insufficiency Study (OACIS) Group
}

\begin{abstract}
Background The hospital stay after acute myocardial infarction (AMI) is still extremely long in Japan and does not correspond with disease severity. The purpose of the present study was to identify a subgroup of AMI patients suitable for early discharge.

Methods and Results A total of 3,739 AMI patients treated with percutaneous coronary intervention (PCI) within $24 \mathrm{~h}$ after onset, who enrolled Osaka Acute Coronary Insufficiency Study between April 1998 and March 2004, were studied. Patients showing no complications within the first 7 days $(n=1,786)$ were considered to be eligible for early discharge. In these patients, multivariate logistic regression analysis selected prior myocardial infarction, left anterior descending coronary artery disease and failed PCI as independent predictors of major complications from day 8 to discharge. Patients without any predictor had significant lower rates of major complications from day 8 to 6 months after discharge than those with 1 or more predictors. Furthermore, in the present study there were no deaths in patients without any predictors.

Conclusion Patients who either do not have serious complications during the first 7 days or any predictor are at very low risk of major complications from day 8 to 6 months after discharge. These carefully selected patients may be suitable for early (day 8) hospital discharge after AMI. (Circ J 2005; 69: 1163-1169)
\end{abstract}

Key Words: Acute myocardial infarction; Hospital stay; Japanese; Percutaneous coronary intervention

I n Western countries, the length of hospital stay after acute myocardial infarction (AMI) has been reduced over the past 3 decades from several weeks to several days $!^{1-16}$ Several factors have lead to the reduction in the length of hospital stay, including the physical and psychological advantages of early mobilization, clinical innovations, and economic pressures to reduce medical costs. In contrast, although the length of hospital stay in Japan has gradually decreased, it is still extremely long, at approximately 4 weeks ${ }^{17-22}$ In a recent study, we showed that the length of hospital stay did not correspond appropriately to the severity of AMI22 These findings suggest that the hospital stay could be reduced without adverse effect for AMI patients in Japan. Furthermore, because the reduction of the

(Received January 31, 2005; revised manuscript received July 25, 2005; accepted July 28, 2005)

Department of Cardiovascular Medicine, Graduate School of Medicine, Osaka University, Suita, *Osaka Rosai Hospital, Sakai, **Ishinkai Yao General Hospital, Yao, Osaka National Hospital, Osaka, ${ }^{\dagger}$ Kansai Rosai Hospital, Amagasaki and ${ }^{\sharp}$ Kawachi General Hospital, Higashi-Osaka, Japan

Mailing address: Hiroshi Sato, MD, Department of Cardiovascular Medicine, Graduate School of Medicine, Osaka University, 2-2 Yamadaoka, Suita 565-0871, Japan. E-mail: satoz@medone.med. osaka-u.ac.jp length of hospital stay has several advantages, it is necessary to adjust the length of hospital stay to correspond to disease severity by selecting candidates for early discharge. The purposes of the present study using a large hospitalbased cohort were: (1) to discover independent predictors of in-hospital major complications; and (2) to identify a subgroup of Japanese AMI patients suitable for early discharge based on these predictors.

\section{Methods}

\section{Patients}

We registered 5,502 consecutive AMI patients in the Osaka Acute Coronary Insufficiency Study (OACIS) from April 1998 to March 2004. Of these patients, 3,739 who were treated with percutaneous coronary intervention (PCI) within $24 \mathrm{~h}$ of onset of AMI were included in this study. A detailed description of the OACIS has been published elsewhere ${ }^{2-25}$ The OACIS is a prospective, multicenter observational study in which 25 collaborating hospitals in Japan recorded demographic, procedural and outcome data, and collected blood samples from patients with AMI. The registry was designed to collect uniform, prospective data on patients with AMI that could be used to assess clinical variables, therapeutic procedures and clinical events, and to 


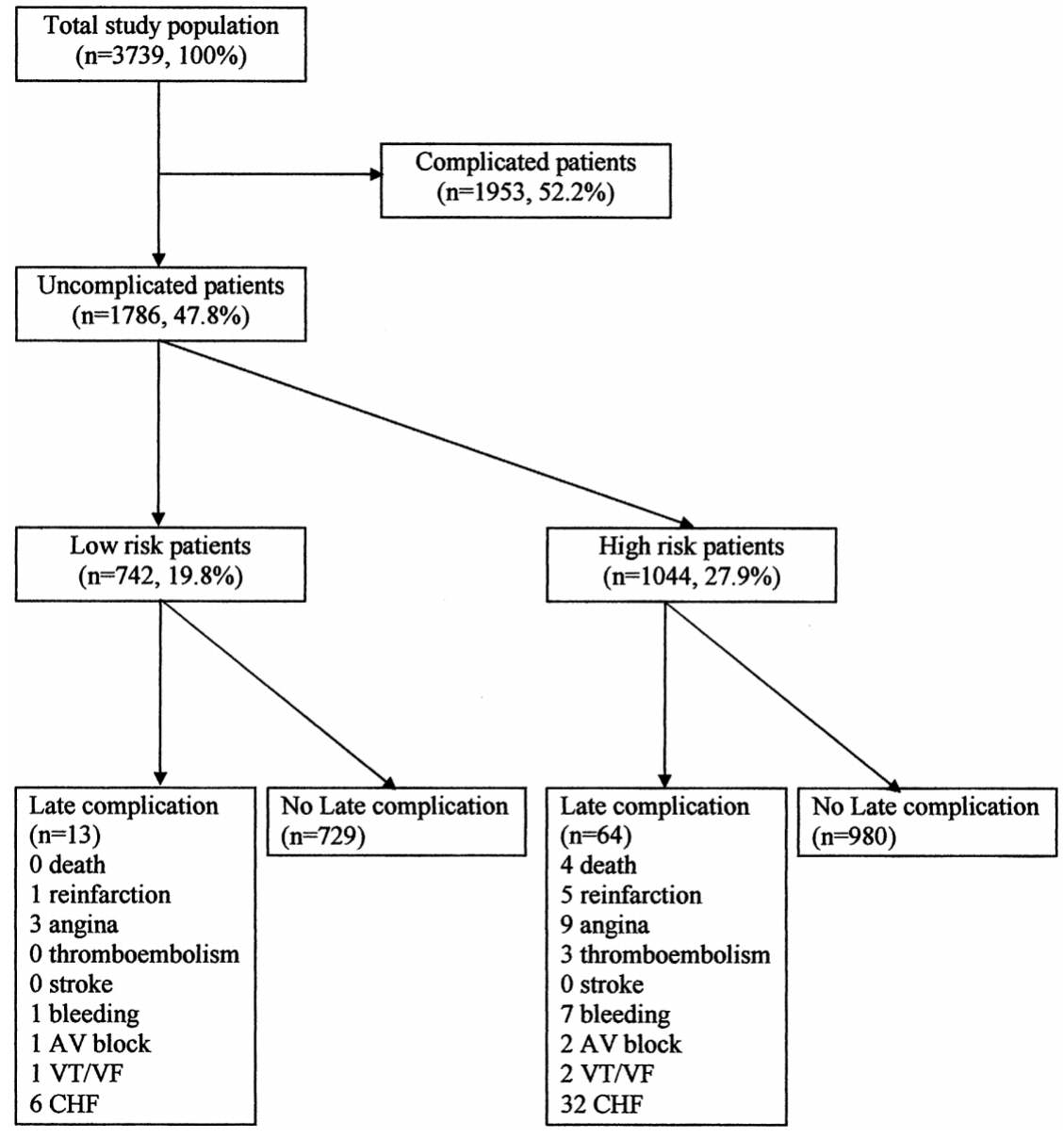

Fig 1. Risk stratification of the total study population. Uncomplicated patients were defined as survivors with the absence of the following conditions or treatments: cardiogenic shock; CHF; reinfarction; angina; ventricular septal perforation; severe mitral regurgitation; cardiac rupture; thromboembolism; stroke; bleeding; VT/Vf; AV block; left main coronary artery disease or the need for coronary artery bypass grafting, percutaneous cardiopulmonary support, intra-aortic balloon pumping, or mechanical ventilation within the first 7 hospital days. Low-risk patients were defined as those who had neither serious complications during the first 7 days nor any independent predictor of late major complications (prior myocardial infarction, left anterior descending coronary artery disease and failed PCI). CHF, congestive heart failure; VT/Vf, ventricular tachycardia/fibrillation; $\mathrm{AV}$, indicates second- or thirddegree atrioventricular; PCI, percutaneous coronary intervention. collect DNA samples from patients with AMI that could be used to investigate whether common genetic variations are involved in the pathogenesis of AMI $2^{2-25}$ The present study protocol was approved by each of the hospitals' ethics committees.

\section{Definitions}

The diagnosis of AMI required the presence of 2 of the following 3 criteria: (1) a clinical history of central chest pressure, pain or tightness lasting for $30 \mathrm{~min}$ or more; (2) typical electrocardiographic changes (ie, ST segment elevation $>0.1 \mathrm{mV}$ in at least 1 standard or 2 precordial leads, ST segment depression $>0.1 \mathrm{mV}$ in at least 2 leads, abnormal Q wave or T wave inversion in at least 2 leads); and (3) an increase in the serum creatine kinase activity by 2 -fold the normal laboratory value. All patients presenting within 1 week after the onset of AMI were registered prospectively, as soon as the diagnosis of AMI had been made.

\section{Data Collection}

Research cardiologists and specialized research nurses recorded data concerning sociodemographic variables, medical history, therapeutic procedures and clinical events during the patient's admission. Information was obtained from the hospital medical records and by direct interview with the patient, family members and the treating physician. After written informed consent for entering the OACIS was given by the patient, all in-hospital data were transmitted to the data collection center located in the Department of Cardiovascular Medicine in Graduate School of Medicine,
Osaka University for processing and analysis.

From patients who were discharged alive, we obtained follow-up clinical data at 3,6 and 12 months after the onset of AMI and annually thereafter. We obtained data concerning subsequent cardiac events through visits to the research outpatient clinic or, in a few instances, by verbal or written contact with the patient's physician, the patient or family members.

\section{Risk Stratification}

Patients with complications within the first 7 days were considered to be ineligible for the early discharge strategy. In the present study, patients without complications within the first 7 days were the first candidates for early discharge. Uncomplicated patients were defined as those who did not have or require the following conditions or interventions: cardiogenic shock; congestive heart failure (CHF); reinfarction; angina; ventricular septal perforation; severe mitral regurgitation; cardiac rupture; thromboembolism; stroke; bleeding; ventricular tachycardia/fibrillation (VT/Vf); second- or third-degree atrioventricular (AV) block; left main coronary artery disease or requiring coronary artery bypass grafting; percutaneous cardiopulmonary support; intraaortic balloon pumping; or mechanical ventilation within the first 7 hospital days. This group of uncomplicated patients was used to determine the factors predicting an uncomplicated course (absence of late major complications) from day 8 to hospital discharge. Late major complications were defined as death as well as CHF, reinfarction, angina, thromboembolism, stroke, bleeding, VT/Vf and 
second- or third-degree AV block.

\section{Statistical Analysis}

Continuous variables between groups were compared using the t-test. Categorical variables were compared using the chi-square test. A multiple logistic regression analysis was used to identify independent predictors of major complications. Analyses of data were performed using SPSS statistical software (SPSS version 11.0, SPSS Japan Inc, Tokyo, Japan). For all analyses, significance was defined as $\mathrm{p}<0.05$.

\section{Results}

\section{Early Complications}

Fig 1 shows risk stratification of the total study population: 1,953 patients had complications within the first 7 days. Table 1 shows the frequency of complications within the first 7 days: 126 (3.4\%) died; 737 (19.7\%) had cardiogenic shock; 1,022 (27.3\%) had CHF; and 92 (2.5\%) had reinfarction. The remaining 1,786 patients were free of early complications. Using our definition of uncomplicated myocardial infarction (MI), 47.8\% $(1,786 / 3,739)$ of the total study population was classified as uncomplicated and therefore eligible for early discharge.

\section{Predictors of Late Major Complications}

Table 2 compares the clinical, angiographic and hospital characteristics between patients with and without late
Table 1 Frequency of Early Complications in the Total Study Population $(n=3,739)$

\begin{tabular}{lc}
\hline \hline & $n(\%)$ \\
\hline Death & $126(3.4)$ \\
Cardiogenic shock & $737(19.7)$ \\
CHF & $1,022(27.3)$ \\
Reinfarction & $92(2.5)$ \\
Angina & $67(1.8)$ \\
Ventricular septal perforation & $18(0.5)$ \\
Severe mitral regurgitation & $20(0.5)$ \\
Cardiac rupture & $45(1.2)$ \\
Thromboembolism & $17(0.5)$ \\
Stroke & $28(0.7)$ \\
Bleeding & $150(4.0)$ \\
VT/Vf & $606(16.2)$ \\
Second- or third-degree AV block & $226(6.0)$ \\
Left main coronary artery & $82(2.2)$ \\
Coronary artery bypass grafting & $60(1.6)$ \\
Percutaneous cardiopulmonary support & $126(3.4)$ \\
Intra-aortic balloon pumping & $839(22.4)$ \\
Mechanical ventilation & $463(12.4)$ \\
Any of the above & $1,953(52.2)$ \\
\hline
\end{tabular}

CHF, congestive heart failure; VT/Vf, ventricular tachycardia/fibrillation; $A V$, atrioventricular.

major complications. Patients with late major complications were less likely to have hyperlipidemia than those without. A history of MI was more likely in patients with late major complications. Patients with late major complications were less likely to be treated with coronary stenting

Table 2 Clinical, Angiographic, and Hospital Characteristics

\begin{tabular}{|c|c|c|c|}
\hline & $\begin{array}{c}\text { No late complication } \\
(n=1,709)\end{array}$ & $\begin{array}{l}\text { Late complication } \\
\qquad(n=77)\end{array}$ & $p$ value \\
\hline \multicolumn{4}{|l|}{ Clinical characteristics } \\
\hline Age, years & 62.9 & 64.9 & 0.122 \\
\hline Male, $\%$ & 79.4 & 80.5 & 0.813 \\
\hline Body mass index, $\mathrm{kg} / \mathrm{m}^{2}$ & 23.9 & 23.3 & 0.175 \\
\hline Diabetes mellitus, \% & 33.1 & 25.3 & 0.160 \\
\hline Hypertension, \% & 53.9 & 51.4 & 0.662 \\
\hline Hyperlipidemia, \% & 48.6 & 33.3 & 0.011 \\
\hline Current smoking, \% & 55.7 & 53.4 & 0.707 \\
\hline Prior myocardial infarction, $\%$ & 10.5 & 19.5 & 0.013 \\
\hline Prior angina pectoris, $\%$ & 26.7 & 31.2 & 0.384 \\
\hline Prior cerebrovascular disease, $\%$ & 8.4 & 8.6 & 0.953 \\
\hline Time from onset to presentation $<6 \mathrm{~h}, \%$ & 74.5 & 78.6 & 0.441 \\
\hline \multicolumn{4}{|l|}{ Angiographic characteristics } \\
\hline \multicolumn{4}{|l|}{ Infarct-related artery, $\%$} \\
\hline Left anterior descending coronary artery & 48.5 & 59.7 & 0.054 \\
\hline Left circumflex coronary artery & 15.9 & 9.1 & 0.107 \\
\hline Right coronary artery & 35.7 & 31.2 & 0.417 \\
\hline Bypass graft & 0.1 & 0.0 & 0.832 \\
\hline Multivessel disease, $\%$ & 25.1 & 31.2 & 0.233 \\
\hline Presence of collateral vessels, \% & 37.3 & 31.1 & 0.280 \\
\hline Preprocedural coronary artery stenosis $\geq 75 \%, \%$ & 99.3 & 98.3 & 0.422 \\
\hline Preprocedural TIMI flow grade $\leq 2, \%$ & 86.3 & 87.5 & 0.773 \\
\hline Thrombolysis, \% & 6.7 & 10.4 & 0.218 \\
\hline Intravenous & 3.9 & 7.8 & 0.092 \\
\hline Intracoronary & 2.8 & 2.6 & 0.899 \\
\hline Stent use, \% & 64.2 & 50.0 & 0.012 \\
\hline Successful PCI*, \% & 93.2 & 83.1 & 0.001 \\
\hline Time to reperfusion $<6 \mathrm{~h}, \%$ & 61.5 & 66.7 & 0.496 \\
\hline \multicolumn{4}{|l|}{ Hospital characteristics } \\
\hline Performance of $\mathrm{PCI}>200$ procedures per year, $\%$ & 83.6 & 77.9 & 0.194 \\
\hline AMI case load $>50$ patients per year, $\%$ & 78.1 & 68.8 & 0.055 \\
\hline
\end{tabular}

*Successful PCI was determined by coronary artery stenosis $\leq 25 \%$ and TIMI flow grade 3 on final angiography.

TIMI, Thrombolysis in Myocardial Infarction; PCI, percutaneous coronary intervention; AMI, acute myocardial infarction. 
Table 3 Multivariate Predictors of Late Major Complications

\begin{tabular}{lccc}
\hline \hline & Chi-square* & $\begin{array}{c}\text { Odds ratio } \\
\text { (95\%confidence interval) }\end{array}$ & p value \\
\hline Age & 1.13 & $1.03(0.98-1.08)$ & 0.287 \\
Male & 0.13 & $0.82(0.27-2.44)$ & 0.716 \\
Body mass index $\geq 25 \mathrm{~kg} / \mathrm{m}^{2}$ & 1.48 & $1.76(0.71-4.38)$ & 0.224 \\
Diabetes mellitus & 0.37 & $1.33(0.53-3.37)$ & 0.542 \\
Hypertension & 0.05 & $0.90(0.37-2.20)$ & 0.824 \\
Hyperlipidemia & 2.64 & $0.46(0.18-1.18)$ & 0.104 \\
Current smoking & 0.20 & $1.24(0.48-3.20)$ & 0.651 \\
Prior myocardial infarction & 7.66 & $2.24(1.27-3.98)$ & 0.006 \\
Prior angina pectoris & 1.41 & $1.78(0.69-4.61)$ & 0.235 \\
Prior cerebrovascular disease & 0.40 & $1.59(0.38-6.62)$ & 0.526 \\
Time from onset to presentation $<6 \mathrm{~h}$ & 0.02 & $0.87(0.27-2.44)$ & 0.883 \\
Left anterior descending coronary artery disease & 8.86 & $4.64(1.69-12.7)$ & 0.003 \\
Multivessel disease & 0.47 & $1.42(0.53-3.83)$ & 0.491 \\
Presence of collateral vessel & 0.06 & $0.89(0.35-2.23)$ & 0.800 \\
Preprocedural coronary artery stenosis $\geq 75 \%$ & 0.61 & $0.44(0.06-3.44)$ & 0.434 \\
Preprocedural TIMI flow grade $\leq 2$ & 0.17 & $1.42(0.27-7.56)$ & 0.166 \\
Thrombolysis & 1.16 & $2.13(0.54-8.44)$ & 0.282 \\
Stent use & 0.66 & $0.69(0.28-1.69)$ & 0.417 \\
Failed PCI & 4.13 & $3.51(1.04-11.8)$ & 0.023 \\
Time to reperfusion $<6$ h $h$ & 0.03 & $1.19(0.13-10.8)$ & 0.874 \\
Performance of PCI $>200$ procedures per year & 2.45 & $0.38(0.12-1.27)$ & 0.117 \\
AMI case load $>50$ patients per year & 1.34 & $2.25(0.57-8.88)$ & 0.248 \\
\hline
\end{tabular}

*Wald's chi-square value. AMI, acute myocardial infarction; PCI, percutaneous coronary intervention; TIMI, Thrombolysis in Myocardial Infarction.

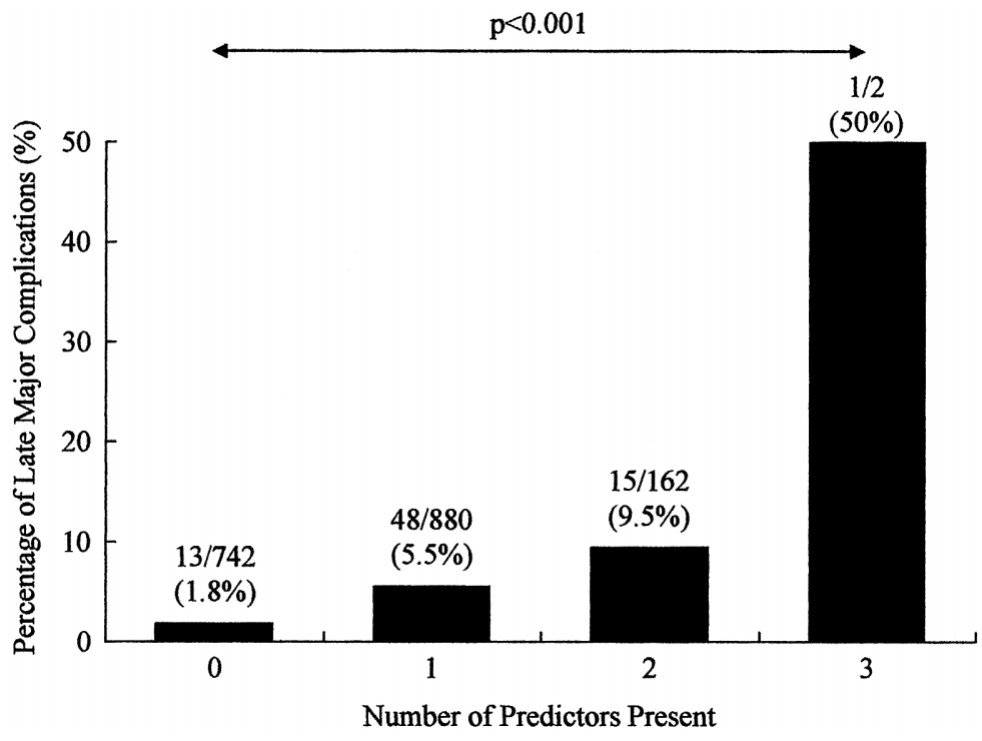

Fig 2. Frequency of late major complications according to the number of predictors. 0 indicates patients with no predictors present; 1 , with 1 predictor; 2 , with 2 predictors; 3 , with 3 predictors. The predictors are prior myocardial infarction, left anterior descending coronary artery disease and failed percutaneous coronary intervention. and had lower rates of successful PCI than those without. Multivariate logistic regression analysis showed that prior MI, left anterior descending coronary artery disease and failed PCI were independent predictors of late major complications (Table 3 ).

\section{Candidates for Early Discharge}

To further identify the subgroup of patients suitable for early discharge, we identified the lowest risk group of late major complications. First, we examined the frequency of late major complications according to the number of predictors in patients without complications during the first 7 days. Fig 2 shows that patients without any independent predictors had significant lower rates of late major complications than those with 1 or more predictors. As shown in Fig 1, 742 patients (19.8\%) were classified as low-risk and
1,044 patients were at high risk of late major complications; $64(6.1 \%)$ of the 1,044 high-risk patients had late major complications: 4 died, 5 had reinfarction, 9 had angina, 3 had thromboembolism, 7 had bleeding, 2 had second- or third-degree AV block, 2 had a VT/Vf and 32 had CHF. However, $13(1.8 \%)$ of the 742 low-risk patients had late major complications: none died, 1 had a reinfarction, 3 had angina, 1 had a bleeding, 1 had a second- or third-degree AV block, 1 had VT/Vf and 6 had CHF. To demonstrate the validity of early discharge in low-risk patients, we compared the frequency of clinical events (death, nonfatal MI, stroke, CHF and revascularization) 6 months after hospital discharge between low- and high-risk patients (Table4). Low-risk patients had a lower risk of 6-month cardiovascular events than high-risk patients. Therefore, patients who had neither complications within the first 7 
Table 4 Six-Month Clinical Events

\begin{tabular}{lccc}
\hline \hline & $\begin{array}{c}\text { Low risk patients } \\
(n=742)^{+}\end{array}$ & $\begin{array}{c}\text { High risk patients }{ }^{\dagger} \\
(n=1,040)^{+}\end{array}$ & $p^{*}$ value \\
\hline Clinical events, \% & 13.2 & 17.6 & 0.012 \\
Death, \% & 0.0 & 0.4 & 0.088 \\
Nonfatal myocardial infarction, \% & 1.1 & 2.5 & 0.027 \\
Stroke, \% & 0.3 & 0.1 & 0.388 \\
CHF, \% & 0.4 & 0.4 & 0.664 \\
Revascularization, \% & 11.9 & 14.2 & 0.154 \\
\hline
\end{tabular}

*Low risk patients were defined as those who had neither serious complications during the first 7 days nor any independent predictor of late major complications. ${ }^{+}$High risk patients were defined as those who had no serious complications during the first 7 days but had 1 or more independent predictors of late major complications. The predictors are prior myocardial infarction, left anterior descending coronary artery disease, and failed PCI. ${ }^{*}$ Sample numbers represent number of patients who were discharged alive in each group. CHF, congestive heart failure; PCI, percutaneous coronary intervention.

days nor any predictor were considered to be suitable for early discharge.

\section{Discussion}

The main finding of the present study is that patients who had neither serious complications during the first 7 days nor any independent predictor of late major complications (prior MI, left anterior descending coronary artery disease or failed PCI) had a very few major complications after day 8 . This result suggests that these carefully selected patients may be suitable for early (day 8) hospital discharge after AMI.

In Western countries, the length of hospital stay after AMI was generally 2-3 weeks in the 1970s! $!^{-3}$ Because of recent clinical innovations, such as the routine use of aspirin, thrombolytic therapy and PCI, which reduce postinfarction complications, early discharge strategies became possible ${ }^{26-28}$ Several randomized studies of whether a more aggressive early discharge strategy was possible in uncomplicated patients with AMI showed that hospital discharge as early as $3-5$ days was safe, feasible and cost effective ? $^{8-12}$ Recently, the hospital stay after AMI has been reduced to about 1 week in Western countries ${ }^{13-16}$

In contrast, although the length of hospital stay has gradually decreased, it is still extremely long, at approximately 4 weeks, in Japan ${ }^{17-22}$ Furthermore, we previously reported that clinical variables, including demographic factors, coronary risk factors, medical history, infarct characteristics, procedures, medication and in-hospital complications, only explained $26 \%$ of the variation in the length of hospital stay, ${ }^{22}$ which suggests that the length of hospital stay does not correspond appropriately with the severity of AMI and that it could be reduced without an adverse effect on the outcome. Furthermore, it is important to reduce the length of hospital stay after AMI to reflect the disease severity in order to improve the physical as well as the psychological status of the patient, as well as reducing medical costs. However, limited data exists on how Japanese AMI patients are identified as suitable for early hospital discharge.

In the present study, we first identified the candidates for early discharge as those without complications within the first 7 days (see Table 1). Next, we identified independent predictors of major complications from day 8 to hospital discharge in these uncomplicated patients, and risk stratification was performed according to these predictors (see Table 3). Third, patients who had neither complications within the first 7 days nor any predictor had the lowest risk of late major complications (see Fig 2). Finally, these patients had very few complications and no deaths within 6 months of discharge (see Table4). Therefore, patients who had neither complications within the first 7 days nor any predictors were considered suitable for early discharge. However, because the purpose of the present study was not to confirm the safety of early hospital discharge in low-risk patients, but to identify such patients, it was impossible to determine whether early hospital discharge strategy was safe. To determine the safety of early hospital discharge in low-risk patients, it is necessary to compare event rates between randomly assigned early discharge groups and late discharge groups. Because previous studies have shown that the 30-day major complication rate in low-risk patients for early discharge is 4-6\%, 1.8\% risk of non-fatal complications was thought to be relatively low and it seems ethically acceptable if the low-risk patients selected in this study were randomly assigned to an early or late discharge group ${ }^{12,29}$

In the present patient population, the mean length of hospital stay was 29.6 days in survivors. Furthermore, the mean hospital stay was 25.8 days in low-risk patients and 26.2 days in high-risk patients. If all low-risk patients were discharged at day 8 , the mean hospital length of stay would be 25.9 days. However, early discharge (day 8 ) of $19.8 \%$ of all patients would reduce the total duration of all hospital stays for AMI by only $13 \%$. The early discharge strategy for low-risk patients has only a limited impact on reduction of medical costs. Therefore, it is necessary to reduce the hospital stay in complicated patients with AMI. The present study could not determine the safety and feasibility of early discharge in complicated patients. A previous randomized controlled study in a Western county has shown that regardless of the presence or absence of complications, early discharge (day 12) had no more adverse events than late discharge (day 19)? Therefore, further study is needed to determine whether early hospital discharge is safe in complicated patients in Japan.

\section{Study Limitations}

The present study was not a randomized controlled study but an observational study. Low-risk patients selected in the present study had only non-fatal complications after day 8 . However, because the patients were actually hospitalized and were under rapid and better management, we consider that they were at very low risk of complications. Therefore, further randomized controlled studies are needed to examine the safety and feasibility of early discharge after AMI in Japan. Previous studies in Western countries of the safety and feasibility of early discharge in patients 
with AMI treated with PCI have reported that an early discharge strategy (days 3-4) can be applied safely to lowrisk patients. ${ }^{10-12}$ Although day 3-4 discharge is thought to be possible for Japanese AMI patients treated with PCI, the present study can not fully answer this question.

\section{Conclusion}

The present study suggests that patients who have neither serious complications during the first 7 days nor any independent predictor of late major complications are at very low risk of major complications after day 8 and that these carefully selected patients may be suitable for early hospital discharge after AMI. These results should be helpful in the development of guidelines, as well as clinical pathways, for management of Japanese AMI patients. However, because the present study has several limitations, randomized controlled studies are needed to examine the safety and feasibility of early discharge after AMI in Japan in order to standardize medical care.

\section{Acknowledgments}

This work was supported by a Grant-in-Aid for Scientific Research $(C)$ (2) (\#15590743) from the Japan Society for the Promotion of Science, Tokyo, Japan and by research funds from the Japan Arteriosclerosis Prevention Fund, Tokyo, Japan.

We thank Mariko Yoneda, Kana Sakatani, Nagisa Yoshioka, Miki Shinkura, Tomomi Miyai, Tomoko Inoue, Akiko Yamagishi, Chikayo Nihashi, Kaori Okada, Shigemi Kohara and Etsuyo Naito for their excellent assistance with data collection.

\section{References}

1. Hutter AM Jr, Sidel VW, Shine KI, DeSanctis RW. Early hospital discharge after myocardial infarction. N Engl J Med 1973; 288: $1141-1144$

2. Wenger NK, Hellerstein HK, Blackburn H, Castranova SJ. Uncomplicated myocardial infarction: Current physician practice in patient management. JAMA 1973; 224: 511-514.

3. Abraham AS, Sever Y, Weinstein M, Dollberg M, Menczel J. Value of early ambulation in patients with and without complications after acute myocardial infarction. N Engl J Med 1975; 292: 719-722.

4. McNeer JF, Wagner GS, Ginsburg PB, Wallace AG, McCants CB, Conley MJ, et al. Hospital discharge one week after acute myocardial infarction. N Engl J Med 1978; 298: 229-232.

5. Hill JD, Hampton JR, Mitchell JR. A randomised trial of home-versus-hospital management for patients with suspected myocardial infarction. Lancet 1978; 1: 837-841.

6. Ahlmark G, Ahlberg G, Saetre H, Haglund I, Korsgren M. A controlled study of early discharge after uncomplicated myocardial infarction. Acta Med Scand 1979; 206: 87-91.

7. Lindvall K, Erhardt LR, Lundman T, Rehnqvist N, Sjogren A. Early mobilization and discharge of patients with acute myocardial infarction: A prospective study using risk indicators and early exercise tests. Acta Med Scand 1979; 206: 169-175.

8. Topol EJ, Burek K, O’Neill WW, Kewman DG, Kander NH, Shea MJ, et al. A randomized controlled trial of hospital discharge three days after myocardial infarction in the era of reperfusion. $N$ Engl $J$ Med 1988; 318: $1083-1088$.

9. Bogaty P, Dumont S, O'Hara GE, Boyer L, Auclair L, Jobin J, et al. Randomized trial of a noninvasive strategy to reduce hospital stay for patients with low-risk myocardial infarction. J Am Coll Cardiol 2001; 37: 1289-1296.

10. Grines CL, Marsalese DL, Brodie B, Griffin J, Donohue B, Costantini $\mathrm{CR}$, et al. Safety and cost-effectiveness of early discharge after primary angioplasty in low risk patients with acute myocardial infarction: PAMI-II investigators: Primary angioplasty in myocardial infarction. J Am Coll Cardiol 1998; 31: 967-972.

11. De Luca G, Suryapranata H, van't Hof AW, de Boer MJ, Hoorntje JC, Dambrink JH, et al. Prognostic assessment of patients with acute myocardial infarction treated with primary angioplasty: Implications for early discharge. Circulation 2004; 109: 2737-2743.

12. Heggunje PS, Harjai KJ, Stone GW, Mehta RH, Marsalese DL, Boura $\mathrm{JA}$, et al. Procedural success versus clinical risk status in determining discharge of patients after primary angioplasty for acute myocardial infarction. J Am Coll Cardiol 2004; 44: 1400-1407.

13. Chen E, Naylor CD. Variation in hospital length of stay for acute myocardial infarction in Ontario, Canada. Med Care 1994; 32: $420-$ 435.

14. Every NR, Spertus J, Fihn SD, Hlatky M, Martin JS, Weaver WD. Length of hospital stay after acute myocardial infarction in the myocardial infarction triage and intervention (MITI) project registry. J Am Coll Cardiol 1996; 28: 287-293.

15. Rogers WJ, Canto JG, Lambrew CT, Tiefenbrunn AJ, Kinkaid B, Shoultz DA, et al. Temporal trends in the treatment of over 1.5 million patients with myocardial infarction in the U.S. From 1990 through 1999: The national registry of myocardial infarction 1,2 and 3. J Am Coll Cardiol 2000; 36: 2056-2063.

16. Bartholomew BA, Harjai KJ, Grines CL, Boura JA, Grines LL, Stone GW, et al. Variation in hospital length of stay in patients with acute myocardial infarction undergoing primary angioplasty and the need to change the diagnostic-related group system. Am J Cardiol 2003; 92: 830-833.

17. Matsui K, Polanczyk CA, Gaspoz JM, Theres H, Kleber FX, Sobashima A, et al. Management of patients with acute myocardial infarction at five academic medical centers: Clinical characteristics, resource utilization, and outcome. J Investig Med 1999; 47: 134140.

18. Matsui K, Fukui T, Hira K, Sobashima A, Okamatsu S, Nobuyoshi $\mathrm{M}$, et al. Differences in management and outcomes of acute myocardial infarction among four general hospitals in Japan. Int J Cardiol 2001; 78: 277-284.

19. Fujiwara K, Hiasa Y, Takahashi T, Yamaguchi K, Ogura R, Ohara Y, et al. Influence of diabetes mellitus on outcome in the era of primary stenting for acute myocardial infarction. Circ J 2002; 66: 800-804.

20. Anzai H, Yoneyama S, Tsukagoshi M, Miyake T, Kikuchi T, Sakurada M. Rescue percutaneous thrombectomy system provides better angiographic coronary flow and does not increase the in-hospital cost in patients with acute myocardial infarction. Circ J 2003; 67: $768-774$.

21. Goto Y, Itoh H, Adachi H, Ueshima K, Nohara R. Use of exercise cardiac rehabilitation after acute myocardial infarction. Circ J 2003; 67: 411-415.

22. Kinjo K, Sato H, Nakatani D, Mizuno H, Shimizu M, Hishida E, et al. Predictors of length of hospital stay after acute myocardial infarction in Japan. Circ J 2004; 68: 809-815.

23. Kinjo K, Sato H, Sakata Y, Nakatani D, Mizuno H, Shimizu M, et al. Impact of smoking status on long-term mortality in patients with acute myocardial infarction. Circ J 2005; 69: 7-12.

24. Ohnishi Y, Tanaka T, Yamada R, Suematsu K, Minami M, Fujii K, et al. Identification of 187 single nucleotide polymorphisms (SNPs) among 41 candidate genes for ischemic heart disease in the Japanese population. Hum Genet 2000; 106: 288-292.

25. Ozaki K, Inoue K, Sato H, Iida A, Ohnishi Y, Sekine A, et al. Functional variation in LGALS2 confers risk of myocardial infarction and regulates lymphotoxin-alpha secretion in vitro. Nature 2004; 429: $72-75$.

26. ISIS-2 (Second International Study of Infarct Survival) Collaborative Group. Randomised trial of intravenous streptokinase, oral aspirin, both, or neither among 17,187 cases of suspected acute myocardial infarction: ISIS-2. Lancet 1988; 2: 349-360.

27. Gruppo Italiano per lo studio della Streptochinasi nell'infarto Miocardico (GISSI). Effectiveness of intravenous thrombolytic treatment in acute myocardial infarction. Lancet 1986; 1: 397-402.

28. Grines CL, Browne KF, Marco J, Rothbaum D, Stone GW, O'Keefe $\mathrm{J}$, et al. A comparison of immediate angioplasty with thrombolytic therapy for acute myocardial infarction: The primary angioplasty in myocardial infarction study group. N Engl J Med 1993; 328: $673-$ 679.

29. Mark DB, Sigmon K, Topol EJ, Kereiakes DJ, Pryor DB, Candela RJ, et al. Identification of acute myocardial infarction patients suitable for early hospital discharge after aggressive interventional therapy: Results from the thrombolysis and angioplasty in acute myocardial infarction registry. Circulation 1991; 83: 1186-1193.

\section{Appendix}

The following institutions and persons participated in the OACIS: Sakurabashi Watanabe Hospital, Osaka, Japan: Fujii K, Ito H; Osaka Police Hospital, Osaka, Japan: Kodama K, Hirayama A; Kansai Rosai Hospital, Amagasaki, Japan: Nagata S, Nanto S, Morozumi T; Ishinkai Yao General Hospital, Yao, Japan: Matsu-ura Y; Osaka General Medical Center, Osaka, Japan: Fukunami M; Osaka Rosai Hospital, Sakai, Japan: Yamada Y, Tanouchi J, Nishino M; Kawachi General Hospital, Higashi- 
Osaka, Japan: Mishima M, Lim YJ; Higashi-Osaka City General Hospital, Higashi-Osaka, Japan: Kijima Y; Osaka National Hospital, Osaka, Japan: Kusuoka H, Koretsune Y, Yasumura Y; Osaka Minami Medical Center, Kawachinagano, Japan: Kinoshita N, Imai K; Osaka Kosei Nenkin Hospital, Osaka, Japan: Sasaki T; Kobe Ekisaikai Hospital, Kobe, Japan: Shimazu T, Fuji H; Yao Municipal Hospital, Yao, Japan: Hoshida S, Umemoto K; Osaka Railway Hospital of West Japan Railway Company, Osaka, Japan: Ezumi A; Kaizuka City Hospital, Kaizuka, Japan: Morita H, Lee JM; Kita-Osaka Hospital, Osaka, Japan: Ogitani N, Ikeda S;
Teramoto Memorial Hospital, Kawachinagano, Japan: Hishida E; Settsu Iseikai Hospital, Settsu, Japan: Akehi N; Kashiwara Municipal Hospital, Kashiwara, Japan: Naka M, Akashi T; Osaka Seamens Insurance Hospital, Osaka, Japan: Kohama A; Saiseikai Senri Hospital, Suita, Japan: Hayashi T, Nakatsuchi Y; Meiwa Hospital, Nishinomiya, Japan: Sugii M; Department of Cardiovascular Medicine, Graduate School of Medicine, Osaka University, Suita, Japan: Otsu K, Yamamoto K, Takashima S, Minamino T; Department of Medical Information Science, Graduate School of Medicine, Osaka University, Suita, Japan: Takeda H, Matsumura Y. 